\title{
Unstable Heterochromatin in Fanconi's Anemia Cells
}

\author{
Yue J. Lin ${ }^{1}$ and Jack R. Edelman ${ }^{2, *}$ \\ ${ }^{1}$ Department of Biological Sciences, Saint John's University, \\ 8000 Utopia Parkway, Jamaica, New York, 11439, U.S.A. \\ ${ }^{2}$ Department of Science, Borough of Manhattan Community College, \\ City University of New York, 199 Chambers Street, \\ New York, New York, 10007, U.S.A. \\ Accepted October 25, 2001
}

\begin{abstract}
Summary Previous investigations in our lab have indicated that cells of patients with Werner's syndrome (WS), a premature aging disorder, contain unstable heterochromatin. That instability results in heterochromatin being sloughed off the surface of chromosomes during high temperature incubation in phosphate buffer. In addition, WS interphase chromocenters are sloughed off into patches and rings during such incubation. Recent investigations in our lab have promulgated the hypothesis that the phenomenon of sister chromatid exchange (SCE) may, likewise, be produced by sloughing off of unstable heterochromatin from the chromosome surface. This hypothesis predicts that cells exhibiting a high SCE frequency, such as Fanconi's anemia (FA), may likewise contain unstable heterochromatin. In order to test this hypothesis, FA cells were subjected to SCE staining conditions, confirming the presence of unstable heterochromatin.
\end{abstract}

Key words Chromocenters, Chromosomes, Fanconi's anemia, Heterochromatin, Sister chromatid exchange, Werner's syndrome.

The premature aging disorder Wernar's syndrome (WS) has been referred to as a chromosomal instability syndrome. For many years, however, the exact nature of that instability had been unknown. We recently found evidence that the instability resided in the heterochromatin content of WS chromosomes (Edelman and Lin 2000), and in that report we concluded that heterochromatin exists as a surface feature or covering of chromosomes.

Subsequent investigations lead us to propose that the phenomenon of sister chromatid exchange (SCE) was formed by translocation of this purported heterochromatin covering from one chromatid to its sister (Edelman and Lin 2001). In that study, evidence indicated that incorporation of bromodeoxyuridine ( $\mathrm{BrdU}$ ) into chromosomes caused the heterochromatin covering to become destabilized; the chromatid incorporating the most BrdU (bifilarly substituted) exhibited more unstable heterochromatin than the chromatid incorporating less BrdU (unifilarly substituted). A mechanism for SCE formation was thereby proposed in which SCE's are produced when sections of the destabilized heterochromatin covering are translocated from the unifilarly BrdU-substituted chromatid to its bifilarly substituted sister chromatid (Edelman and Lin 2001).

This newly proposed mechanism of SCE formation predicts, by extension, that cells with a high frequency of SCE formation should contain destabilized heterochromatin. In order to test this hypothesis, we examined Fanconi's anemia (FA) cells, which are known to have a high SCE frequency (Fujiwara et al. 1984) for the presence of unstable heterochromatin.

*Corresponding author, e-mail: themadprofessor47@hotmail.com 


\section{Materials and methods}

Fanconi's Anemia (FA) cells were obtained from 2 sources. PD4 cells (B cells from a child with FA) were kindly provided by the laboratory of Dr. Grover C. Bagby, Jr., M.D. and Ms. Winifred Keeble of Oregon Health Sciences University. These cells were EBV transformed for immortalization. Cells were incubated in hypotonic solution $(0.075 \mathrm{M} \mathrm{KCl})$ for $10 \mathrm{~min}$ at $37^{\circ} \mathrm{C}$, centrifuged, fixed in Carnoy's solution (3:1 methanol:acetic acid) for $1 \mathrm{~h}$, dropped onto glass slides, air dried, and allowed to age for $1 \mathrm{~d}$.

Slides containing cells from an EBV-immortalized Fanconi lymphocyte cell line (subtype D) were kindly provided by the laboratory of Dr. Alan D'Andrea, M. D., and Dr. Akiko Shimamura, M.D., Ph.D. of the Dana Farber Cancer Institute of Harvard University. These investigators also provided slides containing cells from a non-Fanconi control lymphocyte cell line. All cells had been treated with hypotonic solution, fixed in Carnoy's solution, and air dried onto the slides.

Some slides of each cell type were subjected to the incandescent light exposure method for the analysis of SCE reported previously (Edelman and Lin 1986, 1991) even though cells had not been exposed to bromodeoxyuridine (BrdU). This procedure involved staining with Hoechst 33258 (Sigma), immersion in Sorensen's phosphate buffer at pH 6.8, and Giemsa staining. Some slides treated in this manner were not stained in Hoechst. Light exposure proceeded for 75 min, during which time the temperature of the immersing buffer peaked at $63^{\circ} \mathrm{C}$.

Other slides of each cell type were examined for the presence of unstable heterochromatin using our previously reported high temperature phosphate incubation procedure (Edelman and Lin 2000, 2001). Slides were placed in Coplin jars containing Sorensen's phosphate buffer, pH 6.8, and incubated in a water bath at $63^{\circ} \mathrm{C}$ for $2 \mathrm{~h}$. Slides were then stained in $3 \%$ Giemsa (Gurr's Improved R66) for $15 \mathrm{~min}$, allowed to air dry, and examined for the presence of rings and patches of heterochromatin.

Several slides of each cell type were stained in 3\% Giemsa for $15 \mathrm{~min}$, rinsed, allowed to air dry, and examined. Such slides were not subjected to any of the above treatments.

\section{Results and discussion}

FA is a familial type of aplastic anemia. Patients exhibit numerous mental and physical abnormalities, as well as melanin deposits/patches on skin, nucleated red blood cells, immature white blood cells, increased levels of hemoglobin F, and bone marrow which becomes hypocellular as the disease progresses; inheritance is autosomal recessive (Brown 1993). Powers (1989) reports that FA patients exhibit retarded growth, skeletal abnormalities, albinism and/or hypopigmentation, atrophy of spleen and heart, kidney abnormalities, and many chromosomal breaks which often result in the development of acute leukemias, hepatoma or esophageal carcinoma; males outnumber females by approximately $2: 1$, and most patients die by their mid to late teenage years.

Latt (1981) reported that the yield of metaphases in FA is low, while Weksberg et al. (1979) reported that FA cells have decreased growth rates, increased generation times, and a decreased probability of completing further division after a successful mitosis; these investigators concluded that the decreased growth rate and increased sensitivity (SCE induction) to certain drugs may be due to a defect in the repair and/or replication of DNA.

Our recent investigations indicated that the chromosomal instability in the premature aging disorder Werner's syndrome may be due to unstable heterochromatin (Edelman and Lin 2000). In that investigation, it was found that incubation of Werner's syndrome lymphoblasts in phosphate buffer at $63^{\circ} \mathrm{C}$ for $2 \mathrm{~h}$ causes heterochromatin, in the form of nuclear chromocenters, to slough-off to the cell periphery resulting in rings and patches of heterochromatin, as shown in Fig. 1C. This phenomenon was not observed in control lymphoblasts, in which chromocenters remained intact 


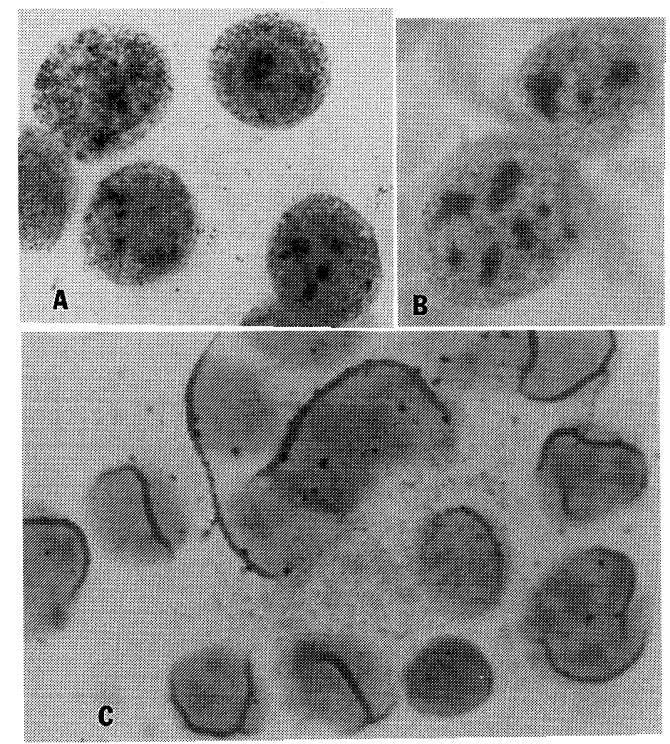

Fig. 1. A) Werner's syndrome interphases stained with Giemsa. Note numerous chromocenters (heterochromatin bodies). B) Fanconi's Anemia interphases stained with Giemsa. Note numerous chromocenters (heterochromatin bodies). C) Werner's syndrome interphases incubated in phosphate buffer at high temperature for $2 \mathrm{~h}$, then stained with Giemsa. Chromocenters (heterochromatin bodies) have been sloughed into rings at the cell periphery, indicating heterochromatin instability in these cells.

after the incubation procedure. As can be seen from Fig. 1A, before incubation, Werner's syndrome chromocenters were intact and did not differ in appearance from those of control cells. Werner's syndrome has long been postulated as a chromosomal instability disorder, although the exact nature of the instability was unknown. The sloughing-off phenomenon had caused us to propose that the nature of the instability was in the heterochromatin, which appeared to function as a cover/surface feature of chromosomes (Edelman and Lin 2000, 2001). In Fig. 1B, it can be noted that heterochromatin/chromocenters are stable in unincubated FA cells as well as unincubated Werner's syndrome cells (Fig. 1A).

When FA cells were incubated in the same manner as Werner's syndrome cells (phosphate buffer at $63^{\circ} \mathrm{C}$ for $2 \mathrm{~h}$, followed by Giemsa staining), the same phenomenon was seen (Fig. 2A, B); heterochromatin/chromocenters were sloughed off to the cell periphery, forming rings and patches. This finding infers that, like Werner's syndrome, FA is a chromosomal instability disorder, with heterochromatin as the source of the instability.

Our rationale for choosing FA cells to test for heterochromatin instability arises out of our recent hypothesis concerning the mechanism of sister chromatid exchange (SCE) formation (Edelman and Lin 2001). In that investigation, we proposed that SCE does not involve breakage and rejoining of entire chromatid sections; rather it involves translocation of the purported heterochromatin cover from the unifilarly BrdU-labeled chromatid to its bifilarly BrdU-labeled sister. In essence, according to our hypothesis, SCE is a surface feature of chromosomes. Since FA cells have a high SCE frequency when exposed to certain substances (Fujiwara et al. 1984), our hypothesis on the mechanism of SCE formation predicts that cells with a high SCE frequency would contain unstable heterochromatin. The results shown in Fig. 2A and B not only pinpoints heterochromatin as 
the source of chromosomal instability in FA, but provides further supporting evidence to our hypothesis concerning the mechanism of SCE formation (Edelman and Lin 2001).

Fig. 2C and D shows FA cells subjected to an SCD staining procedure (Edelman and Lin 1986, 1991), even though the cells were not labeled with BrdU, in order to examine the effects of the staining procedure on heterochromatin stability. Such cells were stained with the fluorochrome Hoechst 33258 before exposure to high intensity incandescent light. From the figures, it can be noted that after the staining procedure, heterochromatin/chromocenters remained intact and stable in appearance. Interestingly, when FA cells were stained using the same procedure, but without Hoechst 33258 staining, much of the heterochromatin/ chromocenters was eroded from cells, giving the cells a 'bleached' or lightly-stained appearance (Fig. 3B). These findings infer that Hoechst staining might stabilize heterochrmatin, and perhaps such stabilization might explain its function in the differential staining (SCD) procedure. A control cell stained with Hoechst

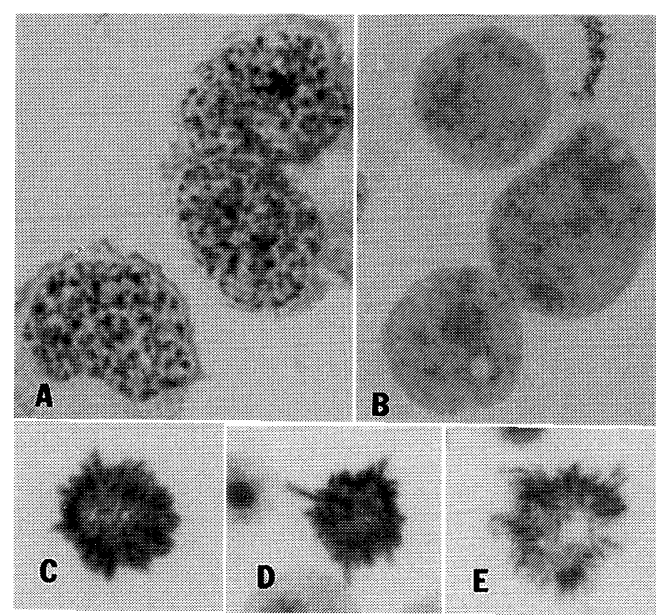

Fig. 3. A) Control cells (non-BrdU labeled) subjected to the SCD staining procedure described in the text. Note stable, intact chromocenters. B) Fanconi's Anemia cells (non-BrdU labeled) subjected to the SCD staining procedure described in the text, but without Hoechst staining. Most of the heterochromatin (chromocenters) has been dissolved out of the cells, indicative of heterochromatin instability in this disorder. Removal of the Hoechst staining procedure reduces heterochromatin stability. C, D, E) Fanconi's Anemia metaphase chromosomes stained with Giemsa. Chromosomes do not separate well, forming clumps in almost all metaphases examined. and subjected to the SCD staining procedure is shown in Fig. 3A; again, heterochromatin/chromocenters remain stable and intact; additional control cells treated with this procedure yielded the same results, even in the absence of Hoechst staining. These findings, taken together, infer that the chromosomal instability of FA cells resides in the heterochromatin.

On a final interesting note, nearly all metaphases seen in FA cells consisted of chromosomes that existed in clumps; they appeared unable to separate, as shown in Fig. 3C-E. This might explain the finding that FA cells exhibit a decreased probability of completing additional divisions after one successful mitosis (Weksberg et al. 1979).

The results of this investigation infer that FA is a chromosomal instability disorder, and like Werner's syndrome, the instability may reside in the heterochromatin, which may function as a covering of chromosomes (Edelman and Lin 2000, 2001). In addition, supporting evidence is provided for our recent hypothesis concerning the role of heterochromatin in the mechanism of SCE formation (Edelman and Lin 2001). Similar studies using cells of other disorders which exhibit high SCE frequencies are currently in progress in our laboratory.

\section{Acknowledgements}

The authors wish to extend their gratitude to the following investigators who were kind enough to provide FA cells, without which this investigation could not be performed: Dr. Grover C. Bagby, Jr., M.D. and Ms. Winifred Keeble, Oregon Health Sciences University; Dr. Alan D'Andrea, M.D. and Dr. Akiko Shimamura, M.D., Ph.D., Dana Farber Cancer Institute of Harvard University. We also wish to thank Dr. George M. Martin, School of Medicine, University of Washington, for Supplying WS cells. 


\section{References}

Brown, B. A. 1993. Hematology-Principles and Procedures. Lea and Febiger, Philadelphia.

Edelman, J. R. and Lin, Y. J. 1986. Simplified differential staining of mouse sister chromatids. Cytobios 46: 147-153.

— and - 1991. The Djungarian hamster, Phodopus sungorus, for the analysis of in vivo sister chromatid exchange. Caryologia 44: 349-354.

— and - 2000. 'Sloughing-off' of heterochromatin in Werner's syndrome cells during high-temperature phosphate incubation. Cytobios 101: 173-185.

- and - 2001. Translocation of unstable heterochromatin as the mechanism of sister chromatid exchange formation: a proposed hypothesis. Cytobios 106: 171-191.

Fujiwara, Y., Kano, Y. and Yamamoto, Y. 1984. DNA Interstrand Cross-linking, Repair, and SCE Mechanism in Human Cells in Special Reference to Fanconi Anemia. In: Tice, R. R. and Hollaender, A. (eds.). Sister Chromatid Exchanges. 25 Years of Experimental Research. Part B. Genetic Toxicology and Human Studies. Plenum Press, New York.

Latt, S. A. 1981. Sister chromatid exchange formation. Ann. Rev. Genet. 15: 11-55.

Powers, L. W. 1989. Diagnostic Hematology. C. V. Mosby Co., St. Louis.

Weksberg, R., Buchwald, M., Sargent, P., Thompson, M. W. and Siminovitch, L. 1979. Specific cellular defects in patients with Fanconi Anemia. J. Cell. Physiol. 101: 311-324. 